\title{
Outline and Index for Chapters III and IV
}

\section{CHAPTER III}

The classification of shapes . . . . . . . . . . 32

1. Introductory comments $\ldots \ldots \ldots \ldots \ldots . \ldots 32$

1. Deep Open Dish and Angular-Rim Dish .. 35

1. Cambered-Rim Dish ................. 38

1. Shallow Dish ...................... 39

1. Cambered-Rim Bowl and Angular-

Rim Bowl ..................... 42

1. Cumbrous Bowl $\ldots \ldots \ldots \ldots \ldots \ldots \ldots \ldots 45$

1. Simple Bottle, Lamp Bottle and Large Bottle ...................47

1. Complex Jar ................... 53

1. Simple Jar ...................57

1. Flaring Cup $\ldots \ldots \ldots \ldots \ldots \ldots \ldots \ldots \ldots$

1. Drum Bottle .....................60 60

1. Rare Ica-tradition shapes of fancy ware $\ldots \ldots 63$

1. Ica 6 utility ware in tomb Th-1 .........64

1. Plain pottery of unknown function in tomb Th-1 .......................64

1. Imitation Chincha shapes at Ica .........64 64

1. Antiques and imitation antiques $\ldots \ldots \ldots 66$

1. Foreign pottery styles in the Late Horizon . . . . . . . . . . . . . . 67

2. Introductory comments $\ldots \ldots \ldots \ldots \ldots 67$

2. Inca .........................67 67

3. Imported $\ldots \ldots \ldots \ldots \ldots \ldots \ldots \ldots 67$

3. Provincial Inca ................68

4. Jar (Rowe, shape a) ...........68 68

4. Plate ......................69 69

5. Group A-1 $\ldots \ldots \ldots \ldots \ldots \ldots \ldots 69$

5. Group A-2 $\ldots \ldots \ldots \ldots \ldots \ldots \ldots \ldots$

5. Group A-3 $\ldots \ldots \ldots \ldots \ldots \ldots \ldots 71$

5. Group B $\ldots \ldots \ldots \ldots \ldots \ldots \ldots \ldots \ldots \ldots$

6. Group B-1 $\ldots \ldots \ldots \ldots \ldots \ldots 71$

6. Group B-2 ............. 73

5. Various Inca-associated Plates .... 73

4. Cooking pot $\ldots \ldots \ldots \ldots \ldots \ldots \ldots 74$

4. Various provincial Inca shapes $\ldots \ldots 75$
5. Jug $\ldots \ldots \ldots \ldots \ldots \ldots \ldots \ldots \ldots \ldots$

5. Dish .................. 76

5. Wide-mouthed jar ............ 76

5. Faceneck jar .............. 76

5. Modeled figure $\ldots \ldots \ldots \ldots \ldots \ldots 76$

2. Chimu ....................... 77

3. Imported $\ldots \ldots \ldots \ldots \ldots \ldots \ldots \ldots \ldots \ldots$

3. Imitation $\ldots \ldots \ldots \ldots \ldots \ldots \ldots \ldots \ldots \ldots$

1. Musical instruments in the Late

Horizon ...................... 78

1. Survivals of Inca-associated shapes of the Late Horizon in Phase 10 ............ 78

1. A unique Ica 10 shape $\ldots \ldots \ldots \ldots \ldots . \ldots 79$

1. Summary ....................... 79 CHAPTER IV

The analysis of decoration . . . . . . . . . . 84

1. Introductory comments $\ldots \ldots \ldots \ldots \ldots 84$

1. Phase 6 ........................ 84

2. Summary .................. 84

2. Analysis in detail $\ldots \ldots \ldots \ldots \ldots \ldots \ldots 85$

3. Smoked blackware .............. 85

3. Plainware .................... 86

3. Painted redware $\ldots \ldots \ldots \ldots \ldots \ldots \ldots 88$

4. Areas covered with design ....... 88

4. Use of colors ................ 88

4. Design arrangement $\ldots \ldots \ldots \ldots .92$

5. Broad bands ............... 92

5. Medium-broad bands .......... 95

5. Upper body bands ............ 95

5. Narrow bands ............... 95

6. Narrow white bands ......... 95

6. Narrow red bands ...........96 96

5. Inner mouth bands ........... 96

5. Lip and cambered-rim bands .....997

5. Handle bands .............. 97

5. Bottle and jar neck decoration ................. 97

5. Special arrangements $\ldots \ldots \ldots .97$ 
6. Shallow Dish ............ 98

7. Half band, flange band, others ................ 98

6. Straight flaring-sided dish .....99

6. Cumbrous Bowl ............. 99

6. Inflected flaring-sided dish ....999

6. Drum Bottle .............. 100

6. Various imitation Chincha and minority shapes $\ldots \ldots \ldots \ldots \ldots 100$

6. Decoration for shapes of aberrant size or other aberrant shape features ............. 100

4. Design patterns and elements ...... 101

5. Diamond and related forms ....... 101

6. Broad band, Large Diamond .... 101 7. Large Diamond with step fillers ....................101

8. Plain and Fish .......... 101

7. Stepless Large Diamond ..... 102

8. Plain and Fish .......... 102

6. Upper body band, Large

Diamond .................102

7. Plain and Fish $\ldots \ldots \ldots \ldots \ldots 102$

7. Composite .............. 102

7. Various ............... 102

6. Various contexts, Large Diamond patterns and their filler elements ................. 102

7. Stepped Large Diamond . . . . 103

6. Small Diamond .............. 103

6. Small Square ............. 104

6. Diamond-shaped composite unit designs ................ 104

7. Diamond elements .......... 104

7. Solid Square elements ........ 104

7. Tailed Square elements ...... 104

7. Bird elements ............ 104

6. Single Diamond unit designs .... 104

7. Stepped Large Diamond . . . . . 104

7. Rotational Fret ............ 105

5. Rectangle and Rectangle Fish .... 105

5. Triangle Fish ................ 105

5. Tailed Square $\ldots \ldots \ldots \ldots \ldots \ldots, 106$

5. Checkerboard ................. 106

5. Wavy Line ................... 106

5. Stripes ................. 106

5. Bird and Fish unit designs ...... 106

5. "Pattée Cross" and cursive figures .................. 106

5. Narrow white-band designs $\ldots \ldots 106$

6. Meander ................ 106
6. "Z" $Z \ldots \ldots \ldots \ldots \ldots \ldots \ldots \ldots \ldots$

6. Stepped " $Z$ " ..............106

6. Solid Triangle ..............106

6. Fret $\ldots \ldots \ldots \ldots \ldots \ldots \ldots \ldots \ldots$

6. Bird .................. 106

5. Modified narrow red-band designs used as narrow white-band decoration ................ 107

5. Narrow red-band designs $\ldots \ldots \ldots 107$

5. Inner mouth-band designs . . . . 107

6. Principal decoration .......... 107

7. Rectangular pattern .........107

7. Triangular pattern ..........107

6. Secondary decoration (fringe band) $\ldots \ldots \ldots \ldots \ldots \ldots \ldots \ldots 107$

7. Pendent Steps ............ 108

7. Pony Fringe $\ldots \ldots \ldots \ldots \ldots 108$

7. Pendent Hooks ............. 108

5. Lip and cambered rim-band designs .................. 108

5. Handle-band designs $\ldots \ldots \ldots \ldots 108$

5. Special Shallow Dish designs $\ldots . .108$

6. Bird Meander.............. 109

6. Various ...................... 109

1. Phase 7 .............................. 109

2. Summary $\ldots \ldots \ldots \ldots \ldots \ldots \ldots \ldots$

2. Analysis in detail $\ldots \ldots \ldots \ldots \ldots \ldots \ldots 109$

3. Smoked blackware ............... 109

3. Plainware.$\ldots \ldots \ldots \ldots \ldots \ldots \ldots \ldots 110$

3. Painted redware $\ldots \ldots \ldots \ldots \ldots \ldots \ldots 110$

4. Design arrangement $\ldots \ldots \ldots \ldots \ldots 110$

5. Broad bands ................. 110

5. Narrow bands .................. 111

5. Unique arrangement.$\ldots \ldots \ldots \ldots 112$

4. Design patterns and elements ..... 112

5. Rectangle ................. 112

5. Diamond ................. 112

5. Cursive figures $\ldots \ldots \ldots \ldots \ldots \ldots 113$

1. Phase 8 ........................... 113

2. Summary $\ldots \ldots \ldots \ldots \ldots \ldots \ldots \ldots \ldots \ldots \ldots$

2. Analysis in detail $\ldots \ldots \ldots \ldots \ldots \ldots \ldots 113$

3. Smoked blackware ............... 113

3. Plainware ...................... 113

3. Painted redware $\ldots \ldots \ldots \ldots \ldots \ldots 113$

4. Areas covered with design ........ 113

4. Design arrangement $\ldots \ldots \ldots \ldots \ldots 114$

5. Traditional broad bands ........ 114

5. Principal bands in upper body-band position, with fluted shoulder or analogue ................. 115

5. Half band, Shallow Dish ........ 115 


\section{Arrangement on Squat Ovoid}

Jars .................... 115

5. Inner mouth-band tradition designs as principal and narrow-band decoration ................. 115

5. Arrangement on faceneck jar .... 116

5. Narrow bands ................ 116

5. Terminal banding . . . . . . . . 117

5. Neck decoration ............. 117

5. Chincha Rim Scallop .......... 117

4. Design patterns and elements ...... 117

5. Diamond ................. 117

5. Rectangle ................. 118

5. Inner mouth-band tradition $\ldots \ldots 118$

5. Line and dot fringe $\ldots \ldots \ldots \ldots 118$

1. Late Horizon styles $\ldots \ldots \ldots \ldots \ldots \ldots \ldots 118$

2. Summary $\ldots \ldots \ldots \ldots \ldots \ldots \ldots \ldots \ldots \ldots \ldots \ldots$

2. Analysis in detail $\ldots \ldots \ldots \ldots \ldots \ldots \ldots, 120$

3. Smoked blackware $\ldots \ldots \ldots \ldots \ldots \ldots 120$

3. A special use of resin paint $\ldots \ldots \ldots \ldots 123$

3. Plainware ................... 123

3. Painted redware and incised

decoration .................... 124

4. Ica 9 decoration ................ 124

5. Introductory comments ........ 124

5. Areas covered with design ....... 125

5. Use of colors and painting techniques $\ldots \ldots \ldots \ldots \ldots \ldots \ldots \ldots \ldots$

5. Design arrangement $\ldots \ldots \ldots \ldots 127$

6. Introductory comments $\ldots \ldots \ldots 127$

6. Principal design bands $\ldots \ldots \ldots 128$

7. Broad-band arrangements $\ldots 128$

8. Three-color pattern ........129

8. Two-color pattern $\ldots \ldots \ldots 130$

8. Special arrangements, minor specialization $\ldots \ldots \ldots \ldots \ldots 130$

9. Flaring Cup $\ldots \ldots \ldots \cdots 130$

9. Drum Bottle $\ldots \ldots \ldots \ldots \ldots 131$

9. Jar $\ldots \ldots \ldots \ldots \ldots \ldots \ldots \ldots \ldots 131$

6. Narrow bands associated with three-color broad-band

arrangment $\ldots \ldots \ldots \ldots \ldots \ldots \ldots 131$

7. Narrow white bands $\ldots \ldots \ldots 131$

7. Narrow red bands $\ldots \ldots \ldots+132$

7. New narrow bands $\ldots \ldots \ldots 132$

6. Upper body bands $\ldots \ldots \ldots \ldots 132$

6. Medium broad-band arrangement with narrow red bands $\ldots \ldots \ldots 132$

6. Inner mouth bands $\ldots \ldots \ldots \ldots 134$

6. Lip bands . . . . . . . . . . 136

6. Handle bands $\ldots \ldots \ldots \ldots \ldots \ldots 136$

6. Neck decoration $\ldots \ldots \ldots \ldots \ldots 136$
6. Special arrangements, major specialization ..............136

7. Shallow Dish $\ldots \ldots \ldots \ldots \ldots 136$

8. Half band ............ 137

8. Other ................. 137

7. Cumbrous Bowl ........... 137

7. Anthropomorphized Footballshaped Jar ................ 137

7. Some Ica-Inca B Large Bottles ............... 138

7. Incised designs on smoked blackware ..............138

5. Design patterns and elements ....139

6. Introductory comments .......139

6. Diamond ............... 139

7. Large Diamond ........... 139

8. Plain ................. 139

8. Composite Large Diamond . 139

8. Composite Large Square ...139

8. Various .............. 140

8. General features ......... 140

8. Fish ................. 140

7. Small Diamond ...........140

7. Diamond-shaped composite unit designs ..............141

8. Solid Square elements ......141

8. Large Diamond Fish elements ............142

8. Incised Diamond Elements .142

6. "Rectangle" ...............142

7. Conservative painted

Rectangle ...............142

8. Plain .................142

8. Rectangle Fish ........... 143

8. Triangle Fish ............143

7. Advanced painted Rectangle ...143

7. Advanced incised Rectangle ...144

6. Tailed Square ..............144

6. Ica Checkerboard ............144

6. Ica Stripes $\ldots \ldots \ldots \ldots \ldots \ldots \ldots 144$

6. Bird unit designs $\ldots \ldots \ldots \ldots \ldots 145$

6. Narrow-band designs . ........145

7. "Z" ...................145

7. Meander $\ldots \ldots \ldots \ldots \ldots \ldots \ldots \ldots \ldots$

7. Black-band design .........145

6. Designs of the inner mouth-band tradition ..................145

7. Rectangular and triangular patterns ................145

7. Fringe-band designs $\ldots \ldots \ldots 146$

8. Pendent Step ...........146

8. Chincha Rim Scallop .......146 


\section{Pendent and Freestanding}

Triangles ............... 146

6. Imitation Inca "Body Scallop" . . 148

4. Ica-Inca decoration $\ldots \ldots \ldots \ldots \ldots 148$

5. Introductory comments ........ 148

5. Areas covered with design ....... 148

5. Use of colors and painting techniques ................ 148

5. Design arrangement $\ldots \ldots \ldots \ldots 150$

6. Inca-style models ........... 150

6. Ica-Inca arrangements on provincial Inca shapes ............. 151

7. Jar (Rowe, shape a) ........ 151

7. Plate ................. 153

7. Various ................. 154

6. Ica-Inca arrangements on Ica-

Inca $A$ shapes $\ldots \ldots \ldots \ldots \ldots 155$

7. Drum Bottle ............. 155

7. Lamp Bottle ............. 155

7. Special vertical panel arrangement ............ 156

7. Special Stripes arrangement .. 159

7. Various adaptations ......... 159

5. Design patterns and elements .... 159

6. Introductory comments ....... 159

6. Cuzco Inca models .......... 159

6. Ica-Inca Figured designs . . . . . 159

7. Tadpole ................ 160

7. Catfish ................. 160

7. Worms and Dots $\ldots \ldots \ldots \ldots 160$

7. Various ................. 161

7. Bird .................. 161

7. Stepped Diamond and Step Fret..$\ldots \ldots \ldots \ldots \ldots \ldots \ldots 1$

7. Secondary unit designs $\ldots \ldots \ldots 162$

8. Provincial Inca insect ...... 162

8. Conservative Ica unit designs ............. 162

8. Humped Animal $\ldots \ldots \ldots 162$

6. Zigzag Band $\ldots \ldots \ldots \ldots \ldots \ldots 162$

6. Checkerboard ............. 162

6. Various associated Ica-tradition designs ................. 163

7. Composite unit designs $\ldots \ldots 163$

7. Rectangle Fish units ........ 164

7. Narrow-band designs ....... 164

4. Decoration related to the Nasca-Inca style $\ldots \ldots \ldots \ldots \ldots \ldots \ldots \ldots \ldots . \ldots \ldots$

4. The decoration of imitation antiques ................... 166

1. Phase $10 \ldots \ldots \ldots \ldots \ldots \ldots \ldots \ldots \ldots \ldots . \ldots \ldots$

2. Summary $\ldots \ldots \ldots \ldots \ldots \ldots \ldots \ldots$
2. Analysis in detail .................. 169

3. Introductory comments ...........169

3. Smoked blackware .............. 170

3. Plainware ................... 170

3. Painted redware ............... 170

4. Areas covered with design ........170

4. Use of colors and painting techniques $\ldots \ldots \ldots \ldots \ldots \ldots \ldots \ldots \ldots 171$

4. Design arrangement $\ldots \ldots \ldots \ldots \ldots 173$

5. Introductory comments ........173

5. Arrangement for neckless vessels ..175

6. Principal bands ............ 175

7. Broad ................. 175

7. Medium broad ............ 175

7. Narrow ................. 175

6. Secondary narrow bands ...... 175

6. Special arrangements, minor specialization ............... 176

7. Flaring Cup ............. 176

7. Cambered-Rim Bowl ........ 177

7. Jars circular in horizontal section ................ 178

5. Arrangement for Complex Jars elliptical in horizontal section $\ldots .178$

6. Broad principal band .........178

7. Vertical panel arrangement $\ldots 178$

7. Horizontal three-band arrangement ............ 179

7. Undivided broad band ......180

7. Modifications around modeled features ................ 180

7. Various adaptations to shape variations ................ 180

5. Arrangement for Simple Bottles and Simple Jars ............. 180

5. Adaptations of the three major categories of design arrangements to non-traditional forms $\ldots \ldots \ldots \ldots 182$

5. Upper body bands ............183

5 . Secondary narrow bands $\ldots \ldots \ldots 184$

6. Narrow white bands ........ 184

6. Narrow red bands .......... 185

6. Narrow black bands ......... 186

5. Inner mouth bands ........... 186

5. Lip and cambered-rim bands . . . . 187

5. Handle bands ............... 187

5. Bottle neck decoration .......... 187

5. Jar neck decoration $\ldots \ldots \ldots \ldots .188$

5. Special design arrangements, major specialization ........... 188

6. Shallow Dish ............. 188

6. Cumbrous Bowl ........... 189 
6. Anthropomorphized Footballshaped Jar ............... 190

6. Survivals of Late Horizon shapes .................. 190

4. Design patterns and elements ..... 190

5. Diamond ................... 190

6. Large Diamond ............. 191

7. Plain .................. 191

7. Composite .............. 192

7. Various patterns .......... 192

7. Large Diamond Fish ....... 192

6. Small Diamond, Small Square,

Half Diamond ............... 193

5. "Rectangle" ................. 195

6. Introductory comments ...... 195

6. Conservative Rectangle ....... 195

7. Zigzag pattern $\ldots \ldots \ldots \ldots \ldots 196$

7. Hook pattern .............. 196

7. Chevron pattern ........... 196

7. Rectangle Fish ............. 196

7. Triangle Fish ............. 198

6. Advanced Rectangle ......... 198

7. Introductory comments ..... 198

7. Diagonal Line ............ 199

7. Crosshatch .............. 199

7. Crosshatch Checkerboard ... 199

7. Serrated Diagonal .......... 199

8. Plain diagonal pattern ...... 199

8. Serrated Diagonal Chevron pattern ...............200

7. Fish and Bird unit designs .... 201

6. Ica-Inca-tradition Rectangle . . . . . 201

6. Revivalistic Ica 6 Rectangle ...... 201

5. Designs of the inner mouth-band tradition ..................... 201

6. Introductory comments ...... 201

6. Inner mouth-band position designs . . . . . . . . . . . . . 202

6. Conservative variants used in other relatively narrow bands $\ldots 202$

7. Narrow principal-band designs ................ 203

7. Secondary narrow red-band designs ............... 204

7. Principles of design transferences, including to upper body-band position ........ 205

6. Innovative variants as principal decoration ................ 206

7. Inner Mouth-Band Checkerboard 206
7. Patterns made with Small Diamond, Small Square and Meander elements ......... 206

7. Enlarged, elaborated inner mouth-band units .......... 207

6. Additional uses of the triangular pattern ............207

5. Various checkerboard patterns ...207

6. Crosshatch Checkerboard and Inner Mouth-Band Checkerboard ................. 207

6. Serrated Diagonal Checkerboard .................. 207

6. Modified Ica-Inca Checkerboard .................... 208

6. Traditional Ica Checkerboard . . . 208

5. Ica Stripes $\ldots \ldots \ldots \ldots \ldots \ldots \ldots 208$

5. Stepped Diamond and Step Fret ... 209

5. Pendent and Freestanding

Triangles $\ldots \ldots \ldots \ldots \ldots \ldots \ldots .210$

5. Unit designs $\ldots \ldots \ldots \ldots \ldots \ldots 211$

6. Introductory comments $\ldots \ldots 211$

6. Primary unit designs $\ldots \ldots \ldots 211$

7. Composite unit designs $\ldots \ldots 212$

8. Freestanding Triangle $\ldots \ldots 212$

8. Diamond-shaped composite unit designs . . . . . . . . 212

7. Stripe Block ............ 214

7. Large Bird ............... 214

7. Humped Animal $\ldots \ldots \ldots \ldots 215$

7. Rectangle Fish ........... 215

7. Various ................ 216

6. Secondary unit designs ....... 216

7. Introductory comments $\ldots \ldots 216$

7. Composite unit designs $\ldots \ldots 216$

8. Diamond-shaped ........ 217

9. Small Diamond elements ...........217

9. Small Square Elements . . 217

9. Solid Square elements . . . 217

8. Other ............. 217

7. Rectangle Fish........... 217

7. Small Bird ...............218

7. Outlined Cross ............ 218

7. Elements of the inner mouthband tradition $\ldots \ldots \ldots \ldots \ldots 218$

7. Meander elements .........218

7. Various ................ 218

7. Zigzag rows of unit designs $\ldots \ldots \ldots \ldots \ldots \ldots \ldots 218$

7. Special arrangements on inner 
rims of Cumbrous Bowls, other shapes ............ 218

8. Pendent Triangle, Pony

Fringe, Pendent Step,

Pendent Hook .219
5. Narrow-band designs...$\ldots \ldots .219$

6. Meander ................219

6. "Z" ...................220

6. Fret ....................220

5. Adaptations of narrow-band designs to other contexts $\ldots \ldots \ldots 220$ 
